\title{
Terapia de substituição renal: qualidade de vida dos pacientes de um hospital de ensino
}

\author{
Kidney replacement therapy: profile \\ of patients in a teaching hospital
}

\author{
Taís Pagliuco Barbosa르, João César Jacon², Maria Cláudia \\ Parro $^{3}$, Adriani Izabel de Souza Moraes ${ }^{4}$, Tatiane Veterin \\ Coneglian $^{5}$, Francielly Aparecida Correa Iembo ${ }^{5}$
}

Resumo

Objetivo: avaliar a qualidade de vida dos pacientes com insuficiência renal crônica no decorrer do tratamento hemodialítico e diferenças em relação ao sexo. Material e Método: estudo descritivo, quantitativo, desenvolvido no setor de Terapia Renal Substitutiva de um hospital escola, constituído por 78 pacientes. Critérios de inclusão: pacientes maiores de 18 anos em tratamento hemodialítico. Foram excluídos pacientes com comprometimento cognitivo. A coleta de dados ocorreu entre junho e julho de 2018, por meio de um questionário com perguntas sobre aspectos sociodemográficos e caracterização clínica dos participantes do estudo. O outro instrumento destinou-se à qualidade de vida, com a utilização do WHOQOL-bref. Os dados foram organizados no Excel $^{\circledR} 2016\left(\right.$ Microsoft $\left.^{\circledR}\right)$. Estudo aprovado pelo Comitê de Ética em Pesquisa envolvendo seres humanos. Resultados: 51,3\% sexo feminino e $48,7 \%$ masculino; $96,2 \%$ não possuiam vínculo empregatício. Verificada a média dos domínios do WHOQOL-bref, sendo constatado que aspectos físicos, psicológicos, relações sociais e meio ambiente obtiveram a média em torno de 3,2 a 3,6, classificando a qualidade de vida média. Conclusão: o estudo identificou uma qualidade de vida média nos pacientes em terapia de substituição renal.

Palavras-chave: Enfermagem; Qualidade de vida; Insuficiência renal.

\footnotetext{
${ }^{1}$ Doutoranda no Programa de Pós-graduação em Enfermagem Fundamental da Escola de Enfermagem de Ribeirão Preto da Universidade de São Paulo (EERP-USP), Ribeirão Preto, São Paulo, Brasil. Docente no Curso de Enfermagem do Centro Universitário Padre Albino (UNIFIPA), Catanduva, São Paulo, Brasil.E-mail: tais.pagliuco@hotmail.com

${ }^{2}$ Doutorando em Enfermagem na Universidade Federal de São Carlos (UFSCar), São Carlos, São Paulo, Brasil. Docente no Curso de Enfermagem do Centro Universitário Padre Albino, Catanduva, São Paulo, Brasil.

${ }^{3}$ Doutorado em Ciências da Saúde pela Escola de Enfermagem de Ribeirão Preto da Universidade de São Paulo, Ribeirão Preto, São Paulo, Brasil. Docente no Curso de Enfermagem do Centro Universitário Padre Albino, Catanduva, São Paulo, Brasil.

${ }^{4}$ Mestranda em Enfermagem na Universidade Federal de São Carlos, São Carlos, São Paulo, Brasil.

${ }^{5}$ Graduação em Enfermagem pelo Centro Universitário Padre Albino, Catanduva, São Paulo, Brasil.
} 


\begin{abstract}
Objective: to evaluate the quality of life of patients with chronic renal failure during hemodialysis and differences in relation to sex. Material and Method: a descriptive, quantitative study, developed in the Renal Replacement Therapy sector of a teaching hospital, consisting of 78 patients. Inclusion criteria: patients over 18 years of age undergoing hemodialysis. Patients with cognitive impairment were excluded. Data collection took place between june and july 2018, through a questionnaire with questions about socio-demographic aspects and clinical characterization of the study participants. The other instrument was aimed at quality of life, using the WHOQOL-bref. The data were organized in Excel ${ }^{\circledR} 2016\left(\right.$ Microsoft $\left.^{\circledR}\right)$. Study approved by the Research Ethics Committee involving human beings. Results: $51.3 \%$ were female and $48.7 \%$ were male, $96.2 \%$ had no employment. The average of the WHOQOL-bref domains was verified, and it was found that physical, psychological, social relations and the environment obtained an average of around 3.2 to 3.6, classifying the average quality of life. Conclusion: the study identified an average quality of life in patients undergoing renal replacement therapy.
\end{abstract}

Keywords: Nursing; Quality of life; Renal insufficiency.

\section{Introdução}

A doença renal crônica (DRC) tem crescido muito nos últimos anos, sendo esta considerada um problema de saúde pública. ${ }^{(1)}$ A alta taxa de morbidade e mortalidade não ocorre somente na população idosa, também está atingindo os mais jovens e trazendo impacto negativo na qualidade de vida destes indivíduos. ${ }^{(2)}$

O tratamento do portador de insuficiência renal crônica (IRC) é realizado através de hemodiálise, que caracteriza-se por um procedimento de substituição da função renal, por meio de uma máquina em que ocorre a remoção de líquidos do corpo quando a função renal do paciente está incapaz de fazê-lo. ${ }^{(3)}$ Esse tratamento é realizado, em média, por três sessões semanais, durante o período de três a cinco horas por sessão, e varia conforme a necessidade do paciente. ${ }^{(4)} \mathrm{O}$ transplante renal bem sucedido é a melhor escolha para eliminar a hemodiálise da rotina dos doentes. ${ }^{(2)}$

Após o doente renal crônico iniciar o tratamento dialítico, muitas mudanças ocorrem em sua vida, sejam familiares ou sociais, pois impedem que o indivíduo realize certas tarefas, aflorando sentimentos de depressão, insegurança, angústia e desânimo, devido às alterações em sua rotina e, consequentemente, na qualidade de vida. ${ }^{(5)}$
Estas mudanças de vida estimulam a adaptação do paciente diante de sua doença e do seu tratamento. ${ }^{(6)}$ Muitos se encontram e se apegam à força divina, à fé e à religião, a fim de melhorar a insegurança e tristeza, além de apoio para retornar às suas vidas sociais. ${ }^{(7)}$

A religião e a espiritualidade têm alcançado mais ênfase na assistência à saúde, uma vez que são percebidas como formas de trazer sentido à vida, paz e esperança para estes indivíduos em meio a tantos acontecimentos. ${ }^{(3)}$

$\mathrm{O}$ afastamento provisório ou definitivo de seu vínculo empregatício é um dos grandes impactos que ocorrem na vida do doente renal crônico, pois, semanalmente, ficam ligados a uma máquina, tendo como consequências dor, moleza, cansaço e câimbra, fazendo com que o mesmo deixe suas atividades cotidianas, empregos e tarefas que exijam muito esforço físico, uma vez que os fatores fisiológicos ficam prejudicados devido ao mecanismo da hemodiálise. ${ }^{(4-8)}$

A prática de atividade física nos pacientes hemodialíticos está diretamente relacionada à melhora na qualidade de vida destes, pois ameniza as alterações causadas pela doença e seu tratamento, e produz grandes efeitos positivos na capacidade funcional do paciente e em sua função muscular. ${ }^{(9)}$ No entanto, mesmo com estes benefícios, alguns 
estudos relatam níveis baixos de atividades físicas realizadas por esses indivíduos, contribuindo para o sedentarismo, aumentando assim a mortalidade nesta população. ${ }^{(10)}$

Um dos aspectos relacionados à mortalidade nesta população é a inatividade física que pode colaborar para a piora na qualidade de vida desses pacientes. Portanto, é primordial verificar as atividades realizadas por esta população, incentivandoos para a prática regular, e possibilitando, assim, melhora da autoestima e qualidade de vida. ${ }^{(10)}$

Outro aspecto importante para a discussão entre este perfil de paciente é em relação à sexualidade, que caracteriza-se pelo equilíbrio básico de um indivíduo, fazendo com que se sinta amado e apoiado por um outro indivíduo, uma vez que traz um sentimento e uma sensação de normalidade, melhorando o seu bem-estar. ${ }^{(11)}$

De acordo com as fragilidades em potencial, que o paciente renal crônico encontra em sua rotina, é fundamental que os profissionais de saúde estejam atentos quanto à forma de avaliação da qualidade de vida desta população, desenvolvendo olhar integral, a fim de orientá-lo e incentivá-lo para melhoria de seu tratamento e qualidade de vida. ${ }^{(5)}$ Portanto, o objetivo deste estudo foi avaliar a qualidade de vida de pacientes com insuficiência renal crônica no decorrer do tratamento hemodialítico e se há diferenças desta qualidade em relação ao sexo.

\section{Material e Método}

Trata-se de um estudo descritivo, transversal, com abordagem quantitativa. A pesquisa foi desenvolvida no setor de Terapia Renal Substitutiva (TRS) de um hospital do município de Catanduva, estado de São Paulo, Brasil. Esse hospital é caracterizado como universitário, de grande porte, que oferece serviços aos pacientes com IRC do próprio município e região. Neste serviço existem máquinas para tratamento hemodialítico, que ocorrem de segunda a sábado, divididos em dois turnos, sendo manhã e tarde. Os pacientes renais crônicos, em média, realizam três sessões de hemodiálise por semana com duração aproximada de quatro horas.
Os participantes do estudo constituíram-se por 78 pacientes renais crônicos submetidos à hemodiálise atendidos em uma unidade de TRS do hospital no período da coleta de dados, de acordo com a rotatividade do local.

Foram considerados critérios de inclusão pacientes maiores de 18 anos com DRC em estágio $\mathrm{V}$ em TRS que realizavam hemodiálise inseridos no programa da instituição, há pelo menos 30 dias, e que aceitaram responder ao questionário. Porém, foram excluídos da pesquisa pacientes que apresentavam comprometimento cognitivo que os impedissem de responder as questões. Os dados foram coletados entre os meses de junho e julho de 2018, totalizando dois meses de coleta.

Para a coleta de dados foram utilizados dois instrumentos. O primeiro foi um questionário contendo questões estruturadas, abordando os aspectos sociodemográficos e caracterização clínica dos participantes do estudo. Em relação aos dados clínicos, foram analisados sexo, idade, tempo em terapia renal substitutiva, presença de fístula arteriovenosa ou cateter, a doença de base que determinou a DRC, reações adversas decorrentes da hemodiálise, entre outras.

$\mathrm{O}$ segundo instrumento destinou-se à coleta de dados referente à qualidade de vida. Utilizou-se o WHOQOL-bref, instrumento testado e validado em várias culturas de 18 países, sob coordenação do World Health Organization Quality of Life Group (WHOQOL Group) da Organização Mundial da Saúde (OMS). ${ }^{(12)}$ No Brasil, a versão abreviada foi traduzida e validada pelo Grupo WHOQOL Brasil, da Universidade Federal do Rio Grande do Sul (UFRGS). ${ }^{(13)}$

O WHOQOL-bref consta de 26 questões, sendo duas questões gerais de qualidade de vida, e as demais 24 questões compõem os 4 domínios: físico, psicológico, relações sociais e meio ambiente, e representam cada uma das 24 facetas que compõem o instrumento original. ${ }^{(14)}$

As questões do WHOQOL-bref possuem quatro tipos de escalas de respostas: intensidade, capacidade, frequência e avaliação, todas graduadas em cinco níveis (1 a 5). Essas escalas são 
do tipo Likert, sendo que a escala de intensidade varia de nada a extremamente; a escala de capacidade varia de nada a completamente; a escala de avaliação de muito insatisfeito a muito satisfeito e muito ruim a muito bom e a escala de frequência varia de nunca a sempre; dispostas de modo que os maiores valores estão relacionados a uma melhor qualidade de vida, à exceção dos itens Q3: dor física, Q4: tratamento e Q26: sentimentos negativos, com pontuação inversa. ${ }^{(13-14)}$

Os dados foram agrupados e organizados em planilha do programa Excel ${ }^{\circledR} 2016$ (Microsoft $^{\circledR}$ ); para o tratamento estatístico realizou-se a análise descritiva das variáveis sociodemográficas, da caracterização clínica dos pacientes em tratamento hemodiaítico e média dos 26 itens do WHOQOLbref, por meio do programa Statistical Package for the Social Sciences (SPSS) ${ }^{\mathrm{TM}}$; utilizou-se o teste de
Mann-Whitney para análise inferencial das médias de qualidade de vida entre homens e mulheres. $\mathrm{O}$ nível de significância adotado para os testes estatísticos foi de $5 \%(\mathrm{p} \leq 0,05)$.

O levantamento dos dados ocorreu após a aprovação do Comitê de Ética em Pesquisa, com parecer n ${ }^{\circ} 2599089$, conforme a Resolução 196/96, que trata de Pesquisa Envolvendo Seres Humanos.

\section{Resultados}

A amostra foi composta por 78 indivíduos, sendo $51,3 \%$ do sexo feminino e $48,7 \%$ do sexo masculino, com idade mediana de 58 anos, sendo a menor idade 15 e a maior 79 . Ao todo, $96,2 \%$ não possuíam vínculo empregatício e 71,8\% já tiveram reação adversa devido à hemodiálise, conforme demonstra a Tabela 1.

Tabela 1 - Características gerais da amostra. Catanduva, São Paulo, Brasil, 2020.

\begin{tabular}{lcc}
\hline Variáveis & n (78) & \% \\
\hline Idade (mediana) & 58 & 55,49 \\
Sexo & & \\
Feminino & 40 & 51,3 \\
Masculino & 38 & 48,7 \\
Reside & & \\
Catanduva & 30 & 38,5 \\
Região & 48 & 61,5 \\
Estado civil & & 15,4 \\
Solteiro(a) & 12 & 60,3 \\
Casado(a) & 47 & 1,3 \\
União estável & 1 & 12,8 \\
Divorciado(a) & 10 & 10,3 \\
Viúvo(a) & 8 & \\
Religião & & 92,3 \\
Sim & 72 & 7,7 \\
Não & 6 & 3,8 \\
Escolaridade & & 60,3 \\
Analfabeto(a) & 3 & \\
Ensino fundamental incompleto & 47 &
\end{tabular}


Continuação

Ensino fundamental completo $\quad 11$

14,1

Ensino médio incompleto

3

3,8

Ensino médio completo

11

14,1

Ensino superior completo

2

2,6

Não sabe

1

1,3

Vínculo empregatício

Sim

3

3,8

Não

75

96,2

Comorbidades

Sim

67

85,9

Não

11

14,1

Tabagista

Sim

10

12,8

Não

68

87,2

Etilista

Sim

2

2,6

Não

76

97,4

Reações adversas decorrentes à hemodiálise

Sim

56

71,8

Não

22

28,2

Praticante de exercícios físicos

Sim

11

14,1

Não

67

85,9

Fonte: os autores.

Sobre qualidade de vida, analisando de for- a percepção da qualidade de vida dos 78 pacientes ma separada seguindo o módulo WHOQOL-bref, ficou com média de 3,45 (Tabela 2).

Tabela 2 - Qualidade de vida geral por meio do instrumento WHOQOL-bref de pacientes em terapia renal substitutiva (TRS). Catanduva, São Paulo, Brasil, 2020.

\begin{tabular}{lcc}
\hline Variáveis & Mín. - Máx. (mediana) & Média \\
\hline Percepção da qualidade de vida & $1-5(4)$ & 3,45 \\
Satisfação com a saúde & $1-5(3)$ & 3,12 \\
\hline
\end{tabular}

Fonte: os autores.

Quando verificada a média dos domínios do

média em torno de 3,2 a 3,6, classificando a qualiWHOQOL-bref aplicados aos pacientes, foi possível constatar que os domínios físicos, psicológicos, relações sociais e meio ambiente obtiveram a Tabela 3. dade de vida, de uma maneira geral, entre nem boa nem ruim e boa, conforme pode se observar na 
Tabela 3 - Descrição da qualidade de vida, segundo os domínios do WHOQOL-bref, de pacientes em terapia renal substitutiva (TRS). Catanduva, São Paulo, Brasil, 2020.

\begin{tabular}{lc}
\hline Domínio & Média \\
\hline 1 (Físico) & $22,84(3,26)$ \\
2 (Psicológico) & $20,63(3,43)$ \\
3 (Relações sociais) & $10,63(3,54)$ \\
4 (Meio ambiente) & $29,00(3,62)$ \\
\hline
\end{tabular}

Fonte: os autores.

Ao se verificar a média geral das questões aplicadas foi obtido valor de 3,45, divergindo mais a Q18, a qual se refere à satisfação com a capacidade de trabalho, obtendo média de 2,41, seguida da Q5, o quão a vida é aproveitada, com média de 2,65 , e a Q21, relacionada à satisfação com a vida sexual, com média de 2,87, ficando estas facetas entre muito pouca e média satisfação (Gráfico 1).

Gráfico 1 - Média de qualidade de vida. Catanduva, São Paulo, Brasil, 2020.

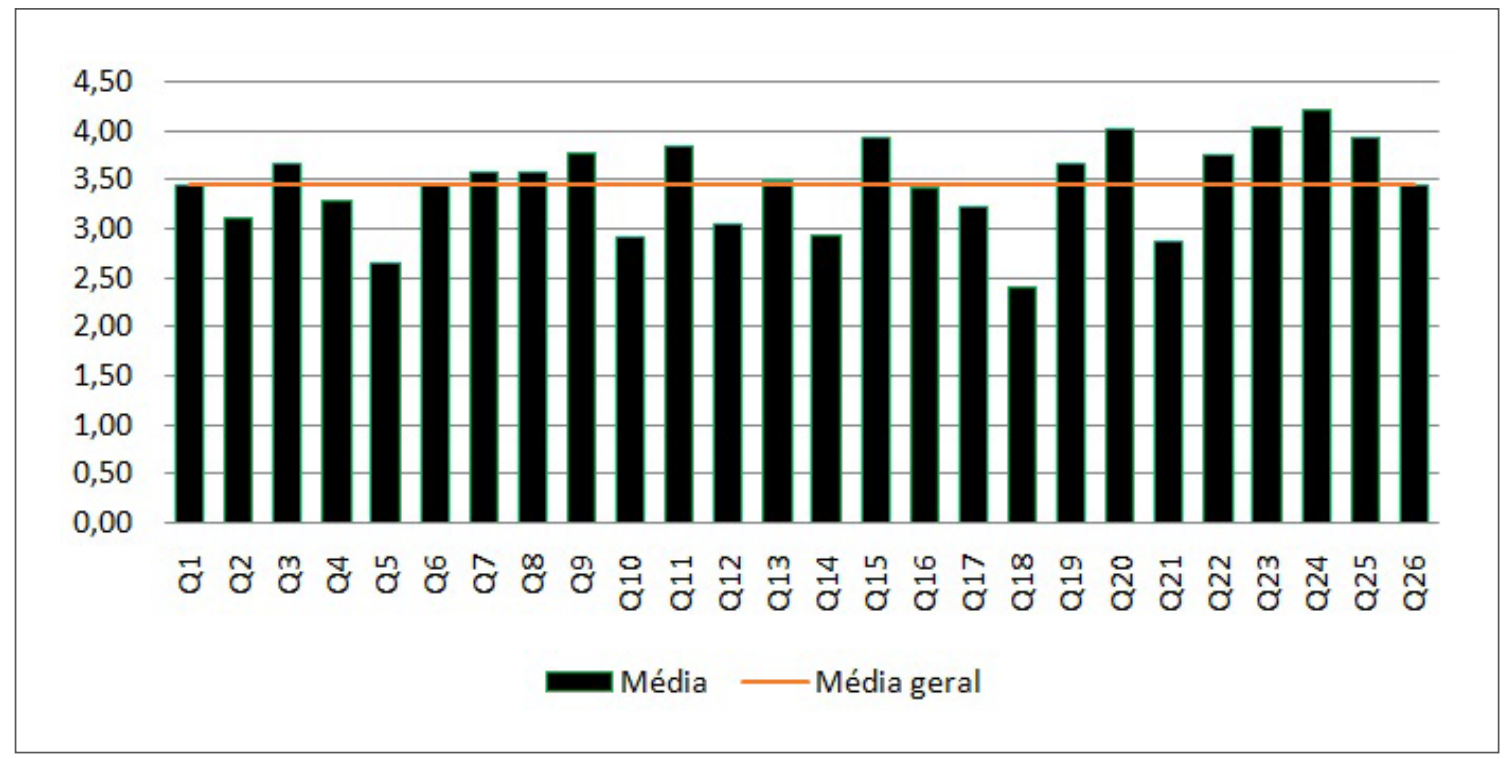

Fonte: os autores.

Quando aplicado o teste não paramétrico de Mann-Whitney, com nível de significância de 5\% para averiguar se há diferença na qualidade de vida entre mulheres e homens, pode-se verificar que não há significância estatisticamente $(p=0,8647)$. Foi possível verificar diferença na qualidade de vida entre homens e mulheres de forma significante estatisticamente apenas na questão Q8, que se refere ao quão seguros(as) se sentem na vida diária, com resultado de $\mathrm{p}=0,0455$.

\section{Discussão}

Neste estudo a média de idade foi de 55,49 anos, idade também observada em outros estudos,

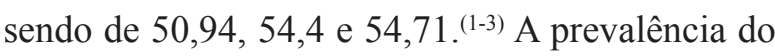
sexo feminino também revelou-se presente no estudo de Pereira, sendo 54,30\%, e de Ferentz, com $50,8 \%{ }^{(15)}$ No entanto, foram encontrados estudos em que o sexo com maior percentual foi o masculino. ${ }^{(1,6)}$ Esse aspecto torna-se relevante quando são 
analisados os impactos distintos para a qualidade de vida entre homens e mulheres, pois nos homens a fragilidade que a doença causa e a necessidade constante de cuidados podem desencadear sentimentos negativos que podem influenciar no cotidiano e na vida social, sobretudo na vulnerabilidade ocasionada pela não redistribuição do poder na residência, em que a mulher assume a função de provedora financeira e de material da família. ${ }^{(16)}$

Entretanto, para a mulher, a DRC e a hemodiálise podem interferir sobre o papel feminino culturalmente instituído na sociedade, em relação ao cuidado da casa e da família, o que pode resultar em prejuízos para a sua qualidade de vida. Os estereótipos de gênero enraizados na sociedade potencializam atitudes baseadas em crenças e valores acerca do papel de homens e mulheres, influenciando nas práticas de saúde e na exposição dos indivíduos a situações de risco. ${ }^{(16)}$

A maioria segue alguma religião, fato este também encontrado no estudo de Oliveira, no qual $73,2 \%$ relataram seguir uma religião. A religião e a espiritualidade estão recebendo cada vez mais ênfase na assistência à saúde, pois podem ser percebidas como maneiras de encontrar sentido para a vida, de ter esperança e estar em paz em meio aos acontecimentos graves, como a doença crônica. ${ }^{(17)}$

Estudiosos que avaliam e documentam os efeitos da espiritualidade e da religião na saúde têm apontado uma relação positiva entre vários aspectos do bem-estar físico e mental, bem como têm considerado que essas podem funcionar como um suporte diante de situações difíceis, de eventos traumáticos e/ou de estresse. Nesse contexto, tanto a espiritualidade como a religiosidade podem ser compreendidas como importantes estratégias de enfrentamento de doenças. ${ }^{(15)}$

Neste estudo, dos 78 pacientes da amostra, $60,3 \%$ são casados, resultado este encontrado em outros estudos, nos quais a maioria dos indivíduos possuíam cônjuges: $57,15 \%, 57,4 \%$ e $69,7 \%$. $^{(15-17)}$ Verificou-se que $87,2 \%$ não possuíam hábito do tabagismo, o que demonstra também no estudo de Jesus, em que $86 \%$ dos entrevistados relataram não serem tabagistas. ${ }^{(15,18)}$
Sabe-se que o tabagismo piora a sobrevida de pacientes em hemodiálise, que diminui $72 \%$ em dois anos e 40\% em seis anos nos fumantes; quando acompanhado de hipertensão arterial, de acordo com alguns autores, esses dados são ainda mais alarmantes. ${ }^{(18)}$

No que diz respeito às comorbidades, $85,9 \%$ possuíam algum tipo, indo de encontro com outro estudo em que $84 \%$ dos pacientes também possuíam comorbidades, e com o estudo de Gonçalves et al., no qual em apenas 9,7\% não havia nenhuma comorbidade. ${ }^{(15,19)}$ Quando verificadas as reações adversas decorrentes da hemodiálise, 71,8\% dos entrevistados já tiveram, fato este encontrado em $57 \%$ dos pacientes em outro estudo. ${ }^{(15)}$

Foi possível verificar que $96,2 \%$ dos pacientes não possuem vínculo empregatício, sendo verificada esta alta porcentagem em outros estudos, os quais demonstraram taxas de 70,2\%, 88,7\% e até de $100 \%$ de indivíduos sem trabalho. ${ }^{(15,19)} \mathrm{E}$ ao se levantar a questão relacionada à prática de atividade física, a maior parte referiu não realizar, $85,9 \%$, sendo vista a falta de adesão a essa prática em outro estudo, com $71 \%$. ${ }^{(19)}$

Ao se questionar a respeito da percepção da qualidade de vida, podendo ser classificada como muito ruim, ruim, nem ruim nem boa, boa e muito boa, neste estudo ela foi classificada entre "nem boa nem ruim" e "boa", com média de 3,45, encontrando em outro estudo a mesma percepção pelos entrevistados: $3,88 .^{(15)}$ Outra faceta foi relacionada à satisfação com a própria saúde, sendo também classificada entre "nem boa nem ruim" e "boa", com média de 3,12 , fato este também observado em outro estudo com média de 3,47. ${ }^{(15-16)}$

Quando verificados os domínios físicos, psicológicos, relações sociais e meio ambiente, estes tiveram média de 3,$26 ; 3,43 ; 3,54$ e 3,62 respectivamente, sendo classificados entre "nem boa nem ruim" e "boa", score esse encontrado semelhante em um estudo de Curitiba, estado do Paraná, no qual foram encontradas as médias 3,$71 ; 3,71 ; 3,65$ e 3,43 para estes domínios. ${ }^{(20)}$

No presente estudo, as limitações foram em relação à forma de classificação do instrumento 
para a coleta de dados, além dos fatores ambientais durante o período da coleta de dados. Foi possível traçar o perfil dos pacientes em hemodiálise em um hospital do noroeste paulista, mostrando a qualidade de vida e rotina destes indivíduos, a fim de direcionar um cuidado de enfermagem mais humanizado de acordo com a necessidade desta população.

O estudo identificou uma qualidade de vida média nos pacientes em terapia de substituição renal. Um aspecto importante foi em relação ao vínculo empregatício e comorbidades, pois muitos apresentam uma doença de base que evoluiu para a insuficiência renal crônica e, devido à rotina de sessões de hemodiálise, a maioria não possui vínculo de trabalho. Então, verifica-se a importância em estudar o perfil desta população, a fim de propor melhorias na qualidade de vida destes pacientes.

\section{Referências}

1 Pereira ERS, Pereira AC, Andrade GB, Naghettini AV, Pinto FK, Batista SR, et al. Prevalence of chronic renal disease in adults attended by the family health strategy. J Bras Neurol. 2016;38(1):22-30.

2 Contente SR, Cavalcante LIC, Silva SSC, Heumann S. Rotina e qualidade de vida de usuários em terapia renal substitutiva. Rev. SPAGESP. 2018;19(2).

3 Sesso RC, Lopes AA, Thomé FS, Lugon JR, Martins CT. Inquérito Brasileiro de diálise crônica 2014. J Bras Nefrol. 2016;38(1):54-61.

4 Guedes KD, Guedes HM. Qualidade de vida do paciente portador de insuficiência renal crônica. Ciênc Saúde. 2012;5(1):48-53.

5 Barcellos RC, Matos JP, Kang HC, Rosa ML, Lugon JR. Comparison of serum creatinine levels in different color/race categories in a Brazilian population. Cad Saude Publica. 2015; 31(7):1565-9.

6 Souza EAS Jr, Trombini DSV, Mendonça ARA, Atzingen ACV. Religião no tratamento da doença renal crônica: comparação entre médicos e pacientes. Rev. Bioét. 2015;23(3):615-22.
7 Pinho NA, Silva GV, Pierin AMG. Prevalência e fatores associados à doença renal crônica em pacientes internados em um hospital universitário na cidade de São Paulo, SP, Brasil. J Bras Neurol. 2015;37(1):91-7.

8 Franceschini N, Gouskova NA, Reiner AP, Bostom A, Howard BV, Pettinger M, et al. Adiposity patterns and the risk for ESRD in postmenopausal women. Clin J Am Soc Nephrol. 2015;10(2):241-50.

9 Ene-Iordache B, Perico N, Bikbov B, Carminati S, Remuzzi A, Perna A, et al. Chronic kidney disease and cardiovascular risk in six regions of the world (ISN-KDDC): a cross-sectional study. Lancet Glob Health. 2016;4(5):e307-19.

10 Marinho AWGB, Penha AP, Silva MT, Galvão TF. Prevalence of chronic renal disease among Brazilian adults: a systematic review. Cad. Saúde Colet. 2017;25(3):379-88.

11 Oliveira APB, Schmidt DB, Amatneeks TM, Santos JC, Cavallet LHR, Brigitte RM. Qualidade de vida de pacientes em hemodiálise e sua relação com mortalidade, hospitalizações e má adesão ao tratamento. J Bras Nefrol. 2016;38(4):411-20.

12 Fukushima RLM, Costa JLR, Orland FS. Atividade física e a qualidade de vida de pacientes com doença renal crônica em hemodiálise. Fisioter Pesqui. 2018;25(3):338-44.

13 The WHOQOL Group. The development of the World Health Organization quality of life assessment instrument (the WHOQOL). In: Orley J, Kuyken W. Quality of life assessment: international perspectives.Heidelberg: Springer Verlag; 1994. p. 41-60.

14 Fleck MPA, Louzada F, Xavier M, Chachamovich E, Vieira G, Santos L, Pinzon V. Aplicação da versão em português do instrumento abreviado de avaliação da qualidade de vida “WHOQOL-bref” Rev. Saúde Pública. 2000;34(2):178-83.

15 Jesus NM, Souza GF, Mendes-Rodrigues C, Almeida OP Neto, Rodrigues DDM, Cunha CM. Quality of life of individuals with chronic kidney disease on dialysis. J. Bras. Nefrol. [Internet]. 2019 [cited 2019 July 2];41(3). 
Available from: https://www.scielo.br/j/jbn/a/ 47L5fY58yBs93xF66wJvDYc/?lang=pt

16 Gomes NDB, Valdevino SC, Leal NPR, Pimenta CJL, Teófilo TJS, Silva CRR, et al. Qualidade de vida de pacientes com insuficiência renal crônica submetidos à hemodiálise. Rev Enferm Atual. 2018;86(24).

17 Oliveira APB, Schmidt DB, Amatneeks TM, Santos JC, Cavallet LHR, Michel RB. Quality of life in hemodialysis patients and the relationship with mortality, hospitalizations and poor treatment adherence. J. Bras. Nefrol. 2016 Dec;38(4):411-20.

18 Gonçalves FA, Dalosso IF, Borba JMC, Bucaneve J, Valerio NMP, Okamoto CT, et al. Qualidade de vida de pacientes renais crônicos em hemodiálise ou diálise peritoneal: estudo comparativo em um serviço de referência de Curitiba-PR.J.Bras.Nefrol.2015;37(4):467-74.

19 Oliveira JF, Marinho CLA, Silva RS, Lira GG. Quality of life of patients on peritoneal dialysis and its impact on the social dimension. Esc. Anna Nery. 2019;23(1):e20180265.

20 Teixeira MGFD, Macedo LOS. Alterações vivenciadas na doença renal crônica: impacto na percepção da autoimagem e sexualidade. Rev Saúde e Desenv. 2016;9(5):166-77.

Recebido em: 21 maio 2020

Aceito em: 18 jan. 2021 
